\title{
A Quadruple Multi-Camera Neutron Computed Tomography System at MLZ
}

\author{
Burkhard Schillinger ${ }^{1, a^{*}}$, Jens Krüger ${ }^{1, b}$ \\ ${ }^{1}$ Heinz Maier-Leibnitz Zentrum and Physics E21, Technische Universität München, \\ Lichtenbergstr.1, 85748 Garching, Germany \\ ảBurkhard.Schillinger@frm2.tum.de , bJens.Krueger@frm2.tum.de
}

Keywords: Detector, Multi-detector, Neutron Imaging, Neutron Computed Tomography

\begin{abstract}
Most neutron imaging systems can accommodate large samples, but recent interest is more focused on small cm-sized samples. With a small field of view for a camera-based detection system, the neutron flux per pixel decreases, and measurement time increases. An earlier approach split a large field of view into smaller fields for individual CT measurements using several rotation axes in the field of view of the camera, but reduces the available amount of camera pixels per tomography field, while many applications require the highest possible resolution even or especially for very small samples. A new approach at MLZ uses a multiple camera system with multiple rotation stages to make better use of the full size of the original neutron beam. With four cameras stacked in a 2x2 matrix, only two rotation stages are required where samples are stacked in an aluminum tube with cutouts above each other. A small, but high quality cooled CMOS camera is employed; joint shielding is built up on the outside of the boxes with lead bricks and PE plates. The first prototype is already working; four more camera boxes are currently in production and will be completed soon. First high-resolution results are shown.
\end{abstract}

\section{Introduction}

Most neutron imaging systems can accommodate large samples of $15-30 \mathrm{~cm}$ size, but recent interest is more focused on small cm-sized samples. In cone-beam X-ray tomography, the beam is emitted from the focal spot of the X-ray tube and illuminates the detector in fixed distance, the flux per pixel remains constant, while the projection ratio and effective resolution can be varied by moving the sample closer to or farther from the focal spot. Since neutron imaging uses a quasi-parallel beam, the neutron flux per area remains constant, and higher resolution can only be achieved with smaller detector pixels. The neutron flux per pixel of the detector decreases with decreasing pixel size, and measurement time increases. Moreover, looking only at small samples with a small detector field of view leaves most of the large neutron beam unused.

There were approaches to split a large field of view into smaller fields for individual CT measurements using a cogwheel-based adapter for the rotation stage [1] or using individual micro rotation stages [2], but this leaves a smaller amount of camera pixels per tomography field, while many applications require the highest possible resolution even or especially for very small samples.Using a super-high resolution camera with 50 megapixel or so would require expensive non-standard optics, increase readout time and data rates, and would be inflexible due to mechanical constraints for setting up samples, and often leave large parts of the field of view unused.

An alternative approach is followed at MLZ, using a multiple camera system with multiple rotation stages to make better use of the full size of the original neutron beam. With four cameras, only two rotation stages are required where samples are stacked in an aluminum tube with cutouts above each other. Cameras are stacked with two on top of each other, and two stacks beside each other.

(c) (1) Content from this work may be used under the terms of the Creative Commons Attribution 3.0 license. Any further distribution of this work must maintain attribution to the author(s) and the title of the work, journal citation and DOI. Published under license by Materials Research Forum LLC. 


\section{A compact high-resolution camera detector}

Most facilities use a high-end CCD or CMOS camera mounted in a large box with a mirror and scintillation screen plus massive lead shielding around the camera against stray gamma radiation and scattered neutrons, often permitting a variation of the field of view by moving the camera within the box. Using such a massive geometry is very inflexible to accommodate more than one camera. Our new approach is to use multiple small camera boxes as compact as possible, with the shielding mounted separately on the outside of the box to allow for flexible installation. The box only contains two lead shields in front and back of the camera, the first surrounding the lens, sideways shielding is only mounted externally. In addition, the part containing the scintillation screen and mirror is designed as a separate item so different sizes can be adapted to the camera. For the compact setup, a small, but high quality cooled CMOS camera type 'ASI178 mm cool' [3] with 3096x2080 pixels and 14 bit ADC is employed.
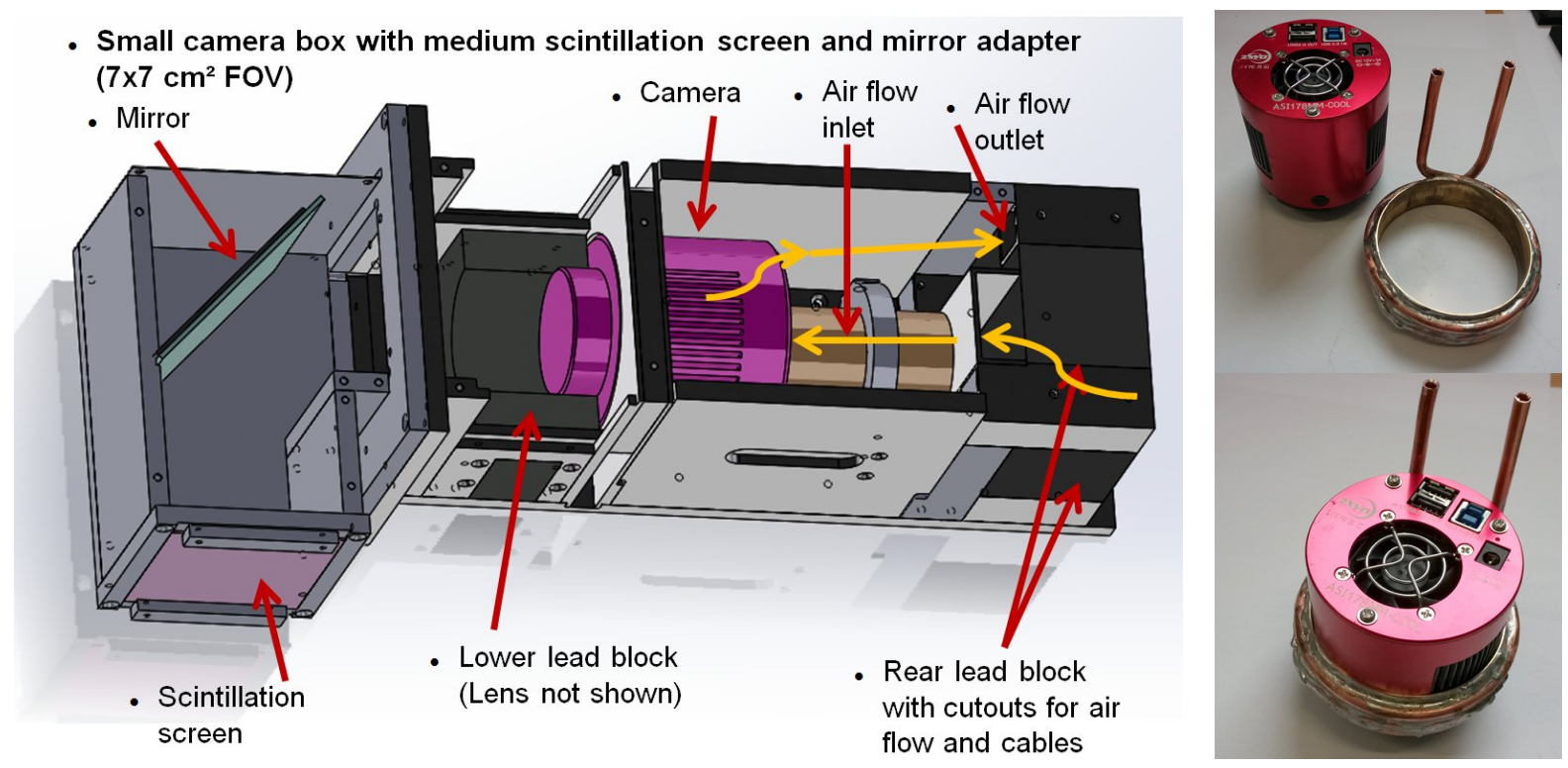

Fig. 1a,b: Schematic drawing of the camera box and photo of the water cooler ring

Fig. 1a shows the schematic drawing of the camera box. A lead block with cutouts for air flow and cables is situated behind the camera, a two-piece lead block surrounds the lens on the front side of the camera. The mirror box is connected by a flange and can be rotated by $180^{\circ}$ to face the other way, or it can be replaced by a mirror box of different size. The selection of the focal length of the C-mount lens allows for a wide variation of the field of view. Once the camera box was mounted within external shielding, the air flow was insufficient for the Peltier cooler built into the camera, so a simple water cooler ring (Fig. 1b) was designed to remove the heat.

\section{The camera stack}

With four boxes stacked in a 2x2 matrix, only two rotation stages are required if samples are mounted in aluminum tubes with cutouts and shelves as displayed in Fig.2 The thin aluminum is nearly transparent for neutrons and does not hinder the tomography of the sample. This kind of sample holder is routinely employed for sequential multi-CT by vertical sample movement at the ANTARES facility. Fig. 2b shows two micro rotation stages as employed in [2].

\section{Software}

The ANTARES neutron CT facility uses a distributed TANGO/Entangle server system with a graphical client system named NICOS, which is described in more detail in [4] in this issue. 
Each physical device is driven by a respective software device. Using multiple rotation devices within one tomography scan has already been implemented for the setup described in [2]. Using more than one detector proved more complicated, since multiple data paths have to be set and used for the multiple detectors. The software generates one path for the measurement with four (or multiple) subdirectories for the respective cameras. For now, only four synchronous identical tomography measurements can be carried out, i.e. with identical angular positions and exposure times.
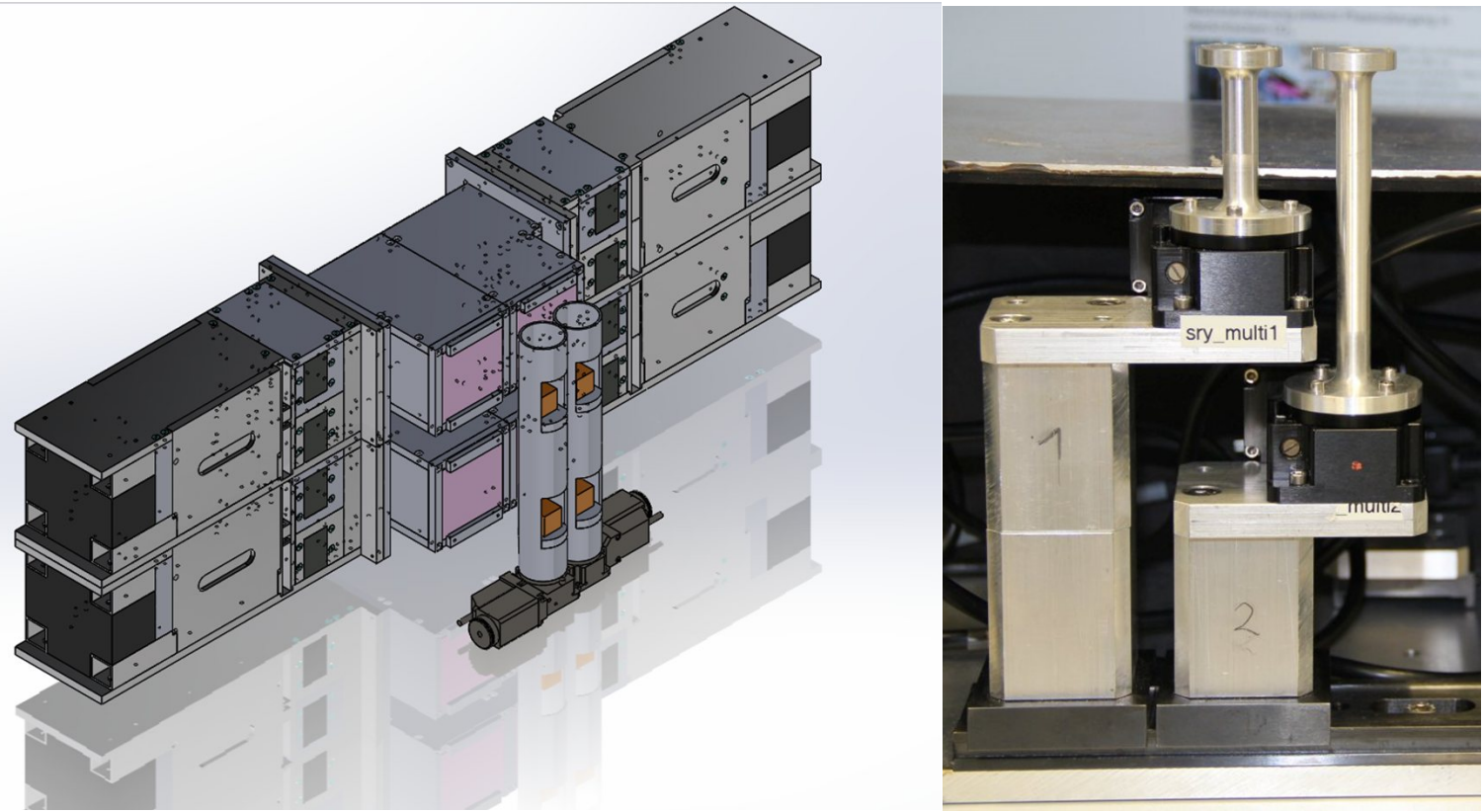

Fig. 2a,b: Schematic drawing of the camera stack and photo of micro rotation stages

\section{High-resolution measurements with the first prototype}

Problems with an external manufacturer who produced the lead blocks far outside specified measures have delayed the completion of the setup, but successful measurements were performed with the first prototype. The quality will be the same for the multiple setup. Fig. 3a shows the prototype mounted on the detector table of the ANTARES facility, with shielding added in Fig. 3b. The standard rotation stage of ANTARES was employed.

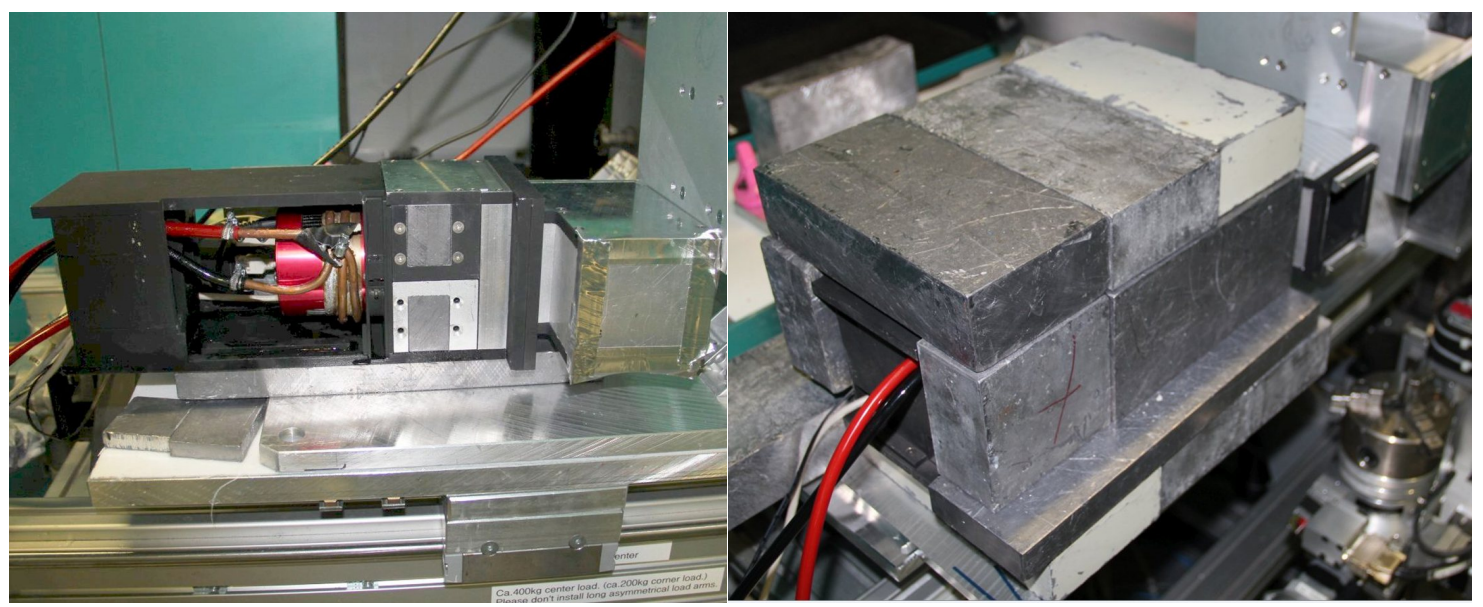

Fig. 3a,b: The prototype detector mounted in the ANTARES facility, bare (Fig.3a) and with added lead shielding (Fig.3b) 


\section{Results}

First measurements were performed with a $50 \mu \mathrm{m} \mathrm{LiF}+\mathrm{ZnS}$ scintillation screen and a $20 \mu \mathrm{m}$ Gadox screen. For each CT, 1278 projections were measured over 360 , with 6 seconds exposure time each, and $9 \mu \mathrm{m}$ effective pixel size. The Gadox screen produced much less light output and thus a weaker signal, but longer exposure times were not possible within the available beam time. Fig. 4 shows reconstructions of a lady's watch. The graininess is attributed to the coarseness of the scintillation screen.

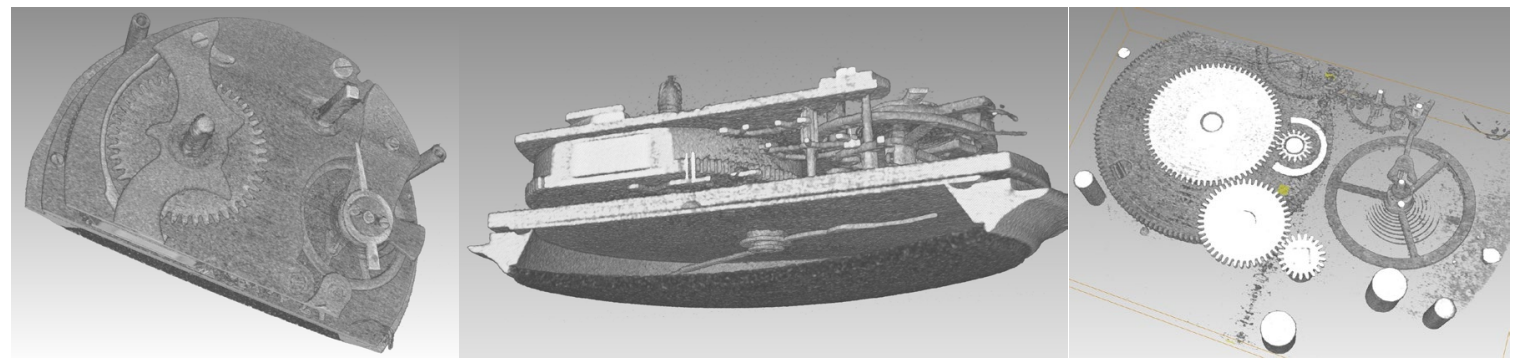

Fig. 4: CT reconstruction of a lady's wrist watch with $Z n S+L i F$ screen at $9 \mu$ m pixel size

Fig. 5a,b shows a photo and reconstruction of a dried hornet, recorded with the same settings. Fig. 6a shows the innards of the hornet recorded with the LiF+ZnS screen, Fig. 6b was recorded with the Gadox screen and shows even higher resolution, but worse statistics.

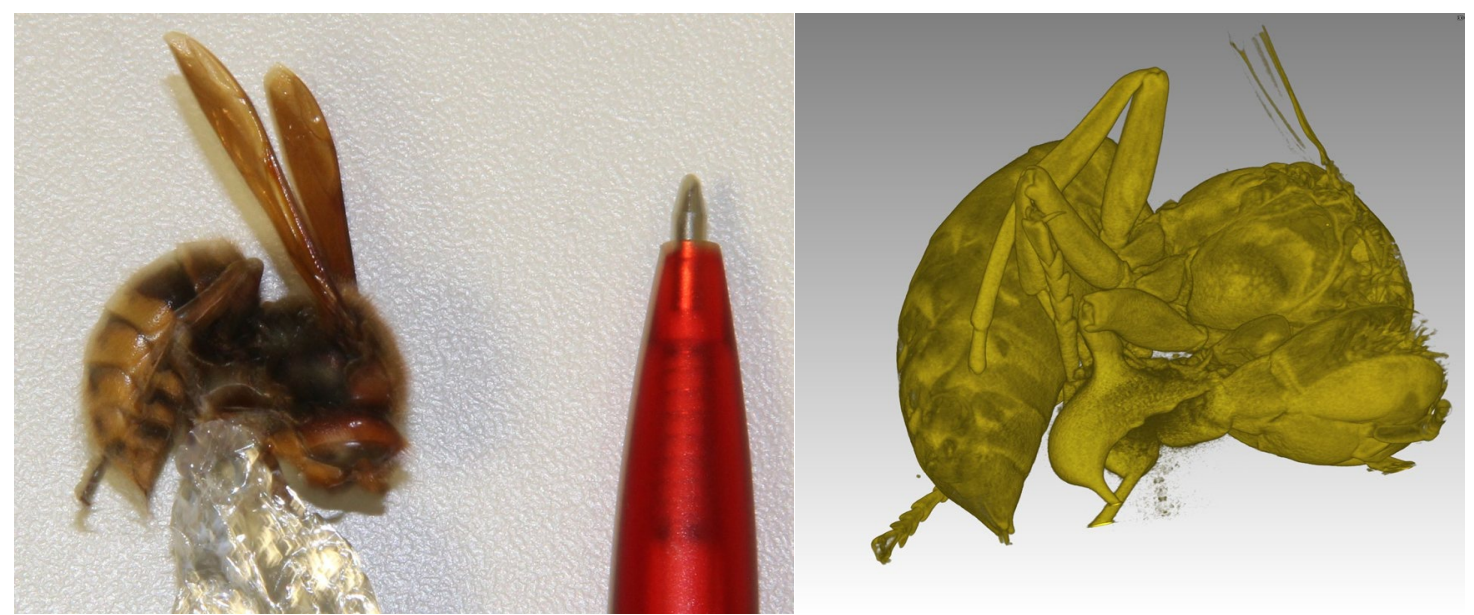

Fig. 5a,b: Photo and CT reconstruction of a hornet with $\mathrm{ZnS}+$ LiF screen at $9 \mu$ m pixel size

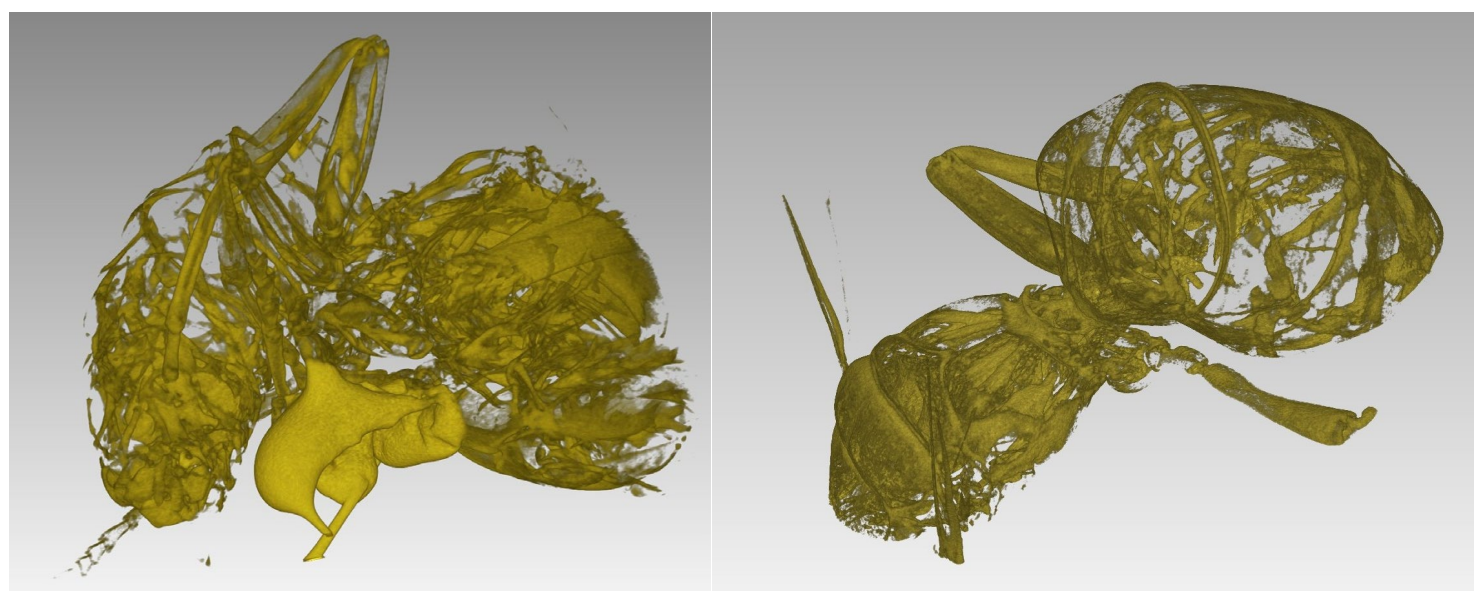

Fig. 6: Photo and CT reconstruction of a hornet with $\mathrm{ZnS}+$ LiF screen (left) and Gadox (right). The Gadox screen gives even higher resolution. 


\section{Conclusions and Outlook}

A 2x2 camera stack is used for multiple synchronous tomography measurements of small samples in a large neutron beam. A compact, but high-quality CMOS detector system was built to be flexibly stacked with external shielding and to be connected to different mirror boxes for different fields of view. The construction of the camera box is currently being worked over for simplification, and the construction drawings and the software described in [4] will be made available to the public in the future. Since the intensity of current neutron sources is limited and cannot be significantly increased, better utilization of available neutron beams can be achieved by synchronous multiple measurements.

\section{References}

[1] P. Trtik, F. Geiger, J. Hovind, U. Lang, E. Lehmann, P. Vontobel, S. Peetermans, Rotation axis demultiplexer enabling simultaneous computed tomography of multiple samples, published online 2016 Apr 18. https://doi.org/10.1016/j.mex.2016.04.005

[2] B. Schillinger, D. Bausenwein, Quadruple axis neutron computed tomography, Physics Procedia 88 ( 2017 ) 196 - 199. https://doi.org/10.1016/j.phpro.2017.06.027

[3] https:// astronomy-imaging-camera.com/product/asi-178mm-cool/

[4] B. Schillinger, A. Craft, J. Krüger, The ANTARES instrument control system for neutron imaging with NICOS/TANGO/LiMA converted to a mobile system used at Idaho National Laboratory, in this issue 